\title{
Optimization of operational conditions for adipate ester synthesis in a stirred tank reactor
}

\begin{abstract}
Esterification of adipic acid and oleyl alcohol in a solvent-free system featuring a stirred tank reactor containing commercially immobilized Candida antarctica lipase B was performed. The process was carried out using an artificial neural network (ANN) trained by the Levenberg-Marquardt (LM) algorithm. The effects of four operative variables, temperature, time, amount of enzyme, and impeller speed, on the reaction yield were studied. By examining different ANN configurations, the best network was found to consist of seven hidden nodes using a hyperbolic tangent sigmoid transfer function. The values of the coefficient of determination (R2) and root mean squared error (RMSE) between the actual and predicted responses were determined to be 1 and 0.0058178 for training and 0.99467 and 0.622540 for the testing datasets, respectively. These results imply that the developed model was capable of predicting the esterification yield. The operative variables affected the yield, and their order of contribution was as follows: time > amount of enzyme > temperature > impeller speed. A high percentage of yield $(95.7 \%)$ was obtained using a low level of enzyme $(2.5 \% \mathrm{w} / \mathrm{w})$, and the temperature, time, and impeller speed were $66.5^{\circ} \mathrm{C}, 354 \mathrm{~min}$ (about $6 \mathrm{~h}$ ), and $500 \mathrm{rpm}$, respectively. A simple protocol for efficient substrate conversion in a solventfree system evidenced by high enzyme stability is indicative of successful ester synthesis.
\end{abstract}

Keyword: Lipase; Adipate ester; Artificial neural network; Stirred tank reactor; Optimization 